\title{
Die Genossenschaft als Nachfolgemodell bei Klein- und Mittelunternehmungen in der Schweiz
}

\author{
Franco Taisch \\ Tizian Troxler \\ Ingrid D'Incà-Keller*
}

\section{Zusammenfassung}

Obschon erfolgreiche Praxisfälle bekannt sind, wird die Genossenschaft bei der Unternehmensnachfolge kaum berücksichtigt. Ausgehend von den Besonderheiten der genossenschaftlichen Rechtsform des Schweizerischen Obligationenrechts zeigt der vorliegende Beitrag thesenartig Konstellationen auf, die für die Genossenschaft als Nachfolgeoption sprechen.

\section{Summary}

In spite of several successful practice cases, business succession concepts take the cooperative form of enterprise hardly into consideration. The present article will, starting from the distinguishing features of a cooperative under the Swiss Code of Obligations, theoretically show constellations in favour of the cooperative form of enterprise as a succession option for a company.

\section{Résumé}

Malgré l'existence connue de certains cas pratiques réussis avérés, la société coopérative n'est guère prise en considération comme option dans les concepts de planification/règlement de l'ordre successoral sociétaire. Partant des spécificités découlant du Code suisse des Obligations pour la forme juridique de la société coopérative le présent rapport, à l'instar d'une thèse, met en avant les situations plaidant pour la société coopérative comme option de planification/règlement successoral.

Prof. Dr. iur. Franco Taisch ist Rechtsanwalt, Ordinarius für Wirtschaftsrecht an der Universität Luzern und Vorsitzender des Direktoriums des IFU | BLI Instituts für Unternehmensrecht und des Kompetenzzentrums für Genossenschaftsunternehmen sowie Präsident der kreisquadrat $\mathrm{GmbH}$, the decision network. Dr. iur. Tizian Troxler ist Advokat, Post-Doc an der Juristischen Fakultät der Universität Basel sowie ehemaliger wissenschaftlicher Mitarbeiter am IFU | BLI Institut für Unternehmensrecht und am Kompetenzzentrum für Genossenschaftsunternehmen, beides an der Universität Luzern. Dott.ssa giur. und MLaw Ingrid D'Incà-Keller ist Doktorandin und wissenschaftliche Mitarbeiterin am IFU | BLI Institut für Unternehmensrecht sowie am Kompetenzzentrum für Genossenschaftsunternehmen, beides an der Universität Luzern. 


\section{Einleitung}

\subsection{Ausgangslage}

Klein- und Mittelunternehmungen (KMU) ${ }^{1}$ stellen zahlenmäßig mit weit über 90 Prozent den größten Anteil an privaten Unternehmen in der Schweiz dar und beschäftigen mehr als zwei Drittel der arbeitenden Bevölkerung. ${ }^{2}$ Als Dienstleistungserbringer, Zulieferer wie auch als Innovatoren von Technologien und Geschäftsmodellen leisten sie überdies einen wichtigen Beitrag zur volkswirtschaftlichen Wertschöpfung und Entwicklung. Aufgrund des Generationenwechsels und des Veränderungsdrucks der Wirtschaft ist in den nächsten fünf Jahren für 20 Prozent der ca. 300.000 meist inhaber- bzw. familiengeführten Klein- und Mittelunternehmungen in der Schweiz eine Regelung ihrer Nachfolge unabdingbar. ${ }^{3}$

Soweit ersichtlich, wird in den einschlägigen Konzepten zur Unternehmensnachfolge die Gründung einer Genossenschaft zur Weiterführung des Unternehmens respektive die Übertragung des Unternehmens an eine Genossenschaft in der Schweiz kaum in Betracht gezogen. Es existieren aber immerhin einige teils wirtschaftlich bedeutende Beispiele, die auf einem genossenschaftlichen Ansatz basieren: ${ }^{4}$

- Erinnert sei etwa an die Entstehungsgeschichte des heutigen Migros-Genossenschafts-Bundes (MGB), dem größten Detailhandelsunternehmen in der Schweiz: Im Jahr 1925 gründete Gottlieb Duttweiler die Migros Aktiengesellschaft (AG) mit dem Ziel, „mehr Umsatz dank weniger Gewinn zu machen

1 Die Terminologie erfolgt in Anlehnung an die Erhebung des Bundesamtes für Statistik, Marktwirtschaftliche Unternehmen und Beschäftigte nach Größenklassen, Betriebszählung 2008, abrufbar unter: http://www.bfs.admin.ch/bfs/portal/de/index/the men/06/02/blank/key/01/groesse.html. Demnach gelten als Klein- und Mittelunternehmungen Unternehmen mit weniger als 250 Beschäftigten, unabhängig von Wirtschaftssektor und Branche. Der Begriff Beschäftigte basiert auf dem Vollzeitäquivalent von $41 /$ 3 Wochen zu 40 Arbeitsstunden pro Monat.

2 Vgl. Bundesamt für Statistik (Fn. 1), abrufbar unter: http://www.bfs.admin.ch/bfs/ portal/de/index/themen/06/02/blank/key/01/groesse.html.

3 Vgl. Halter, F./Kammerlander, N.: KMU Nachfolge - Quo Vadis?, Unsichere Zeiten - ist jetzt der falsche Moment?, in: Bericht 1/2012, Bern 2012, S. 6; Frey, U./Halter, F./Zellweger, T.: Nachfolger gesucht! Empirische Erkenntnisse und Handlungsempfehlungen für die Schweiz, Zürich 2005, S. 12.

4 Auch im internationalen Vergleich finden sich Beispiele, wie etwa die Übernahme des Kohlebergwerks Tower Colliery durch die Mitarbeiter (vgl. Eisen, A.: Unternehmensnachfolge: Eine genossenschaftliche Alternative, in: Dialog 1/2003, S. 36) oder die Rettung des „Wirtshus“ vor dem „Gasthaussterben“ durch die Riefenberger Bürger im Vorarlberg (Vgl. Vorarlberger Nachrichten vom 15.08.2013, abrufbar unter: http://www. vorarlbergernachrichten.at/lokal/vorarlberg/2013/08/14/riefensberger-burger-retten-ihrwirtshus.vn.). 
$[\ldots]]^{“ 5}{ }^{5}$ Er reduzierte die Detailhandelsmarge um die Hälfte und vertraute darauf, dass die höheren Umsätze die Einbußen mehr als nur wettmachen würden. ${ }^{6}$ Diese (Discounter-)Idee führte die Migros AG zum Erfolg. Im Jahr 1941 begann Duttweiler damit, die neun und später zwölf regionalen Tochtergesellschaften der Migros AG in Genossenschaften umzuwandeln. In einem Stiftungsakt schenkte er eingetragenen Kunden und Mitarbeitern Anteilscheine mit einem Nominalwert von je 30 Schweizer Franken. Damit ging ein Genossenschaftskapital von insgesamt rund 4 Mio. Schweizer Franken in das formelle Eigentum von damals 75.540 Mitgliedern über. Die regionalen Genossenschaften schlossen sich später zum heutigen Migros-Genossenschafts-Bund (MGB) zusammen. Die Umwandlung wurde als rein steuerliche Maßnahme sowie als Umgehung des damaligen Filialverbots verschrien. ${ }^{7}$ Unabhängig davon dürfte die primäre Zielsetzung des kinderlosen Duttweilers aber die langfristige Sicherung seines Lebenswerks sowie das soziale und kulturelle Engagement gewesen sein. ${ }^{8}$

- Im Jahr 2013 gründeten die Mieter eines Stadtzürcher Gebäudes eine Genossenschaft, welche die entsprechende Liegenschaft, deren Verkauf an einen externen Investor drohte, übernahm. Der Hauptgrund für den Erwerb lag in der Sicherung preiswerter Wohnräumlichkeiten. ${ }^{9}$ Ferner wurde durch die genossenschaftliche Organisationsform der Fortbestand der Hausgenossenschaft beim Auszug eines Mitglieds gewährleistet ${ }^{10}$ sowie die Blockierung des Projekts durch Einzelne verhindert. ${ }^{11}$

$5 \quad$ Vgl. http://www.migrosmagazin.ch/migros-welt/neues-aus-der migros/artikel/wiedutti-geschichte-schrieb.

6 Vgl. Fn. 5.

7 Es handelte sich einerseits um die im Jahre 1938 geschaffene Ausgleichssteuer (von welcher Genossenschaften ausgenommen waren) und andererseits um den dringlichen Bundesbeschluss betreffend Filialverbot (von welchem Genossenschaften ebenfalls ausgenommen waren). Vgl. Welskopp, T.: Ein ,unmöglicher Konzern“?, Die Migros als Gegenstand der modernen Unternehmensgeschichte, in: Girschik, K./Ritschl, A./ Welskopp, T. (Hrsg.): Der Migros-Kosmos, Zur Geschichte eines aussergewöhnlichen Schweizer Unternehmens, Baden 2003, S. 22-24; Munz, H.: Das Phänomen Migros, Die Geschichte der Migros-Gemeinschaft, Zürich 1973, S. 122-125.

$8 \quad$ Vgl. Munz, H. (Fn. 7), S. 121-123; Taisch, F.: Genossenschaftsgruppen und deren Steuerung, Zürich/St. Gallen 2009, Rz 109.

9 Vgl. Källin, A.: Wie aus Mietern Hausbesitzer werden, in: NZZ vom 11.09.2013, S. 18 .

10 Der Austritt steht dem Genossenschafter gemäss Art. 842-844 OR grundsätzlich frei. Das Schweizerische Bundesgericht toleriert allerdings im Interesse des Fortbestandes der Genossenschaft gewisse Einschränkungen des Austrittsrechts. Vgl. MeierHayoz, A./Forstmoser, P.: Schweizerisches Gesellschaftsrecht, mit neuem Recht der GmbH, der Revision und der kollektiven Kapitalanlagen, 11. Aufl., Bern 2012, § 19 N 69.

11 Das demokratische Kopfstimmprinzip (Art. 885 OR) führt in Verbindung mit der statutarischen Beschränkung der Vertretungsmöglichkeit dazu, dass es bei Unternehmen 
- Ein weiteres aktuelles Beispiel findet sich sodann im schweizerischen Toggenburg. Die Dorfbewohner schlossen sich zu einer Genossenschaft zusammen und retteten die örtliche Arztpraxis. ${ }^{12}$ Dabei stand nicht die Beteiligung am Gewinn im Vordergrund, sondern die Sicherung der lokalen medizinischen Grundversorgung. ${ }^{13}$ Diese Grundausrichtung ermöglichte es zudem - ähnlich wie bei einer Wohnbaugenossenschaft - Praxisräumlichkeiten zu attraktiven Preisen zu vermieten. Dies führte in der Folge zur Niederlassung von Ärzten im Dorf, wodurch die lokale Grundversorgung, als Ziel der Genossenschaft, auch erreicht werden konnte. $^{14}$

- Eine genossenschaftliche Nachfolgelösung wurde schließlich auch im Rahmen der Privatisierung des zuvor öffentlich-rechtlich organisierten Skilifts Tenna umgesetzt. ${ }^{15}$

\subsection{Fragestellung und Vorgehen}

Die genannten Beispiele erlauben die Annahme, dass ganz besondere Interessen und Zielsetzungen einer genossenschaftlichen Nachfolgelösung zugrunde liegen dürften. ${ }^{16}$ Im Folgenden sollen diese spezifischen Interessen und Ziele thesenartig dargelegt werden. Dabei bietet es sich an, bei den Besonderheiten der genossenschaftlichen Rechtsform anzusetzen und davon ausgehend jene Konstellationen herzuleiten, die für die Verwendung der Genossenschaft sprechen. ${ }^{17}$

in der Rechtsform der Genossenschaft schwierig ist, über die Generalversammlung Einfluss zu nehmen. Zwar ,[...] kommt jedem Genossenschafter das gleiche Stimmrecht zu, aber eben das gleich geringe [...]“. Meier-Hayoz, A./Forstmoser, P. (Fn. 10), § 19 N 79 a.

12 Vgl. http://www.genossenschaftspraxis.ch, Genossenschaft, Hintergrund; Rohner, A.: Die Genossenschaftspraxis - aus dem Dorf und für das Dorf, in: PrimaryCare 20/ 2012, S. 394-395.

13 Mit der Genossenschaft dürfen, von Gesetzes wegen, keine rein dividendenstrebigen Zwecke verfolgt, sondern es muss ex lege die Förderung bestimmter Interessen der Mitglieder oder eine gemeinnützige Tätigkeit angestrebt werden. Vgl. Forstmoser, P./ Taisch, F./Troxler, T./D'Incà-Keller, I.: Der Genossenschaftszweck - gestern und heute, in: REPRAX 2/2012, S. 6.

${ }_{14}$ Vgl. Rohner, A. (Fn. 12), S. 394-395. Vgl. zum Ganzen auch Jungmeister, A./Thi, A./Halter, F.: Die Wahl der Rechtsform, Die Genossenschaft als Option im Nachfolgeprozess, in: Stiftung KMU Next (Hrsg.): Bericht Nr. 3/2013, S. 15 f.

15 Vgl. hierzu Jungmeister, A./Thi, A. /Halter, F. (Fn. 14), S. 19 f., m. w. N.

16 Vgl. hierzu auch Jungmeister, A./Thi, A. /Halter, F. (Fn. 14), S. 21-23, m. w. N.

17 Vgl. zu diesem methodischen Ansatz auch Taisch, F./Jungmeister, A./Troxler, T./ D'Incà-Keller, I.: Differenzierungsmerkmale der Unternehmensplattform Genossenschaft und ihr strategisches Potenzial, Interdisziplinäres Forschungsprojekt des IFU | BLI 
Nicht Gegenstand des vorliegenden Beitrags bildet demgegenüber die konkrete Gestaltung der Genossenschaft sowie die Transaktionsstruktur. Bei Letzterem stellen sich grundsätzlich die gleichen technischen, familien-, erb-, gesellschaftsund steuerrechtlichen Fragen, wie bei der Strukturierung jeder anderen Unternehmensnachfolge auch. ${ }^{18}$ Es kommen dabei sowohl die auf dem Kaufvertrag basierenden Transaktionsformen Asset Deal und Share Dea ${ }^{19}$ als auch die auf dem Fusionsgesetz beruhenden Formen Fusion, Umwandlung, Abspaltung und Vermögensübertragung ${ }^{20}$ infrage. Welche Variante die optimale Transaktionsstruktur darstellt, hängt vom Einzelfall ab und kann nicht pauschal beantwortet werden. Gleiches gilt im Übrigen für die konkrete Gestaltung der Genossenschaft.

\section{Besonderheiten der Genossenschaft des Schweizerischen Obligationenrechts}

\subsection{Personenbezogenheit und demokratische Organisation}

Wesensprägend und urtypisch ist unbestreitbar die Personenbezogenheit der Genossenschaft. ${ }^{21}$ Nicht die Mehrheit des Kapitals bestimmt und lenkt, sondern die Mehrheit der Mitglieder. Diese Personen- statt Kapitalbezogenheit widerspiegelt sich besonders in der demokratischen Struktur der Genossenschaft. ${ }^{22}$ Die Mitglieder ,[...] regeln ihre Angelegenheiten selbständig und eigenverantwortlich $[\ldots]^{\text {“23 }}$ durch unabhängige und freiheitliche Entscheidungen in den dafür vorgesehenen Gremien und Formen (Generalversammlung, Delegiertenversammlung, Urabstimmung). Den Kern dieser Struktur stellt das zwingende Kopfstimmprinzip (,one man one vote“) dar, ${ }^{24}$ was eine proportionale Verknüpfung von Kapital und

Instituts für Unternehmensrecht, Universität Luzern, Kompetenzzentrum für Genossenschaftsunternehmen, Zwischenbericht 2012, Luzern 2012, S. 17-20.

18 Vgl. Halter, F./Schröder, R.: Unternehmensnachfolge in der Theorie und Praxis, Das St. Galler Nachfolgemodell, Bern/Stuttgart/Wien 2012, S. 95.

19 Vgl. Herzog, T.: Kauf und Verkauf von KMU in der Schweiz, Ein Leitfaden, Zürich/St. Gallen 2013, S. 55.

20 Vgl. hierzu Meier-Mazzucato, G.: Entgeltliche Unternehmensnachfolge von KMU mit Schwerpunkt steuerliche Aspekte, Bern 2009, S. 312.

21 Vgl. Reymond, J.-A./Trigo Trindade, R.: Die Genossenschaft, in: Meier-Hayoz, A. (Hrsg.): Schweizerisches Privatrecht, Handelsrecht, VIII/V, Basel 1998, S. 3.

22 BSK OR II-Baudenbacher, N 5 zu Art. 828 OR. Vgl. insbes. auch Art. 828, 854 f., 879-882, 885 OR.

23 Schmidt, M.: Unternehmenskultur: Integration des kulturtheoretischen Forschungsansatzes in die Betriebs- und Genossenschaftslehre, Diss. Wien 1995, S. 84.

${ }^{24}$ Ausnahmen bestehen für Delegierte in der Delegiertenversammlung. Dazu Reymond, J.-A./Trigo Trindade, R. (Fn. 21), S. 141. 
Stimmrecht ausschließt und somit die Dominanz der mitunter eindimensionalen Interessen der Kapitalgeber erschwert.

Gewiss lassen sich demokratische Strukturen und selbst das Kopfstimmprinzip mit anderen Gesellschaftsformen des Obligationenrechts - ja selbst mit der Aktiengesellschaft - auf freiwilliger Basis verwirklichen. Bei der Genossenschaft ist dies aber nicht lediglich fakultativ möglich, sondern gesetzlich zwingend. ${ }^{25}$ Genau genommen besteht das Wesensmerkmal „Demokratie“ somit nicht nur darin, dass eine demokratische Struktur besteht, sondern auch darin, dass die Genossenschaft davon nicht abweichen darf. ${ }^{26}$ Die Genossenschaft eignet sich somit dann als Rechtsträger, wenn Gesellschaftsbeschlüsse durch die Mehrheit aller Gesellschafter - und nicht bloß durch die Mehrheitskapitalgeber - mitgetragen werden sollen.

\subsection{Förderung bestimmter Interessen statt reine Dividendenstrebigkeit}

In ihrer gesetzlichen Grundkonzeption ist die Genossenschaft als förderwirtschaftliche, dienende oder hilfswirtschaftliche Einrichtung auf die unmittelbare Förderung der Mitglieder oder - bei gemeinnütziger Tätigkeit - Dritter ausgerichtet. ${ }^{27}$ Eine nur indirekte Verschaffung materieller oder ideeller Vorteile in Form von Geldleistungen allein genügt nicht. ${ }^{28}$ Die Genossenschaft soll ihre Mitglieder unmittelbar durch das Beschaffen von Produkten und das Erbringen von Dienstleistungen , ,...] zu günstigen Bedingungen und / oder Preisen nahe den Kosten $[\ldots]^{629}$ fördern. ${ }^{30}$ Diese Grundausrichtung verhindert bereits konzeptionell, dass Gewinne lediglich angestrebt werden, um sie als Dividende auszuschütten. ${ }^{31} \mathrm{Mehr}$ noch, die Verfolgung rein gewinnstrebiger ${ }^{32}$ - oder besser dividendenstrebiger ${ }^{33}-$

25 Vgl. Art. 885 OR.

26 In diesem Zusammenhang sind auch die Urabstimmung und die Delegiertenversammlung zu berücksichtigen. Vgl. Forstmoser, P./Taisch, F./Troxler, T./D'Incà-Keller, I. (Fn. 13), S. 31f.

27 BSK OR II-Baudenbacher, N 15 zu Art. 828 OR; BGE 80 II 71, E. 1; CHKCourvosier, N 5 zu Art. 828 OR. Vgl. Art. 828 OR und Art. 86 lit. b HRegV.

28 Vgl. Meier-Hayoz, A./Forstmoser, P. (Fn. 10), § 19 N 17-19.

29 Münkner, H.-H.: Organisiert euch in Genossenschaften!, Weiterführende Überlegungen zu Stéphane Hessel's Streitschrift «Empört euch!», Marburg 2013, S. 14.

${ }_{30}$ CHK-Courvoisier, N 6 zu Art. 828 OR.

31 Vgl. auch die Bestimmungen zur Berechnung und Verteilung eines allfälligen Reingewinns (Art. 858-861 OR).

32 Gewinnstrebigkeit im Rechtsinne liegt nur dann vor, ,[...] wenn das (einzige oder hauptsächliche) Ziel einer Geschäftstätigkeit darin besteht, Ertrag zu erzielen, um diesen als Dividende auszuschütten, und den Mitgliedern dadurch einen wirtschaftlichen Vorteil in der neutralen Form von Geld zu verschaffen [...]". Forstmoser, P./Taisch, F./Troxler, T./ D'Incà-Keller, I. (Fn. 13), S. 7. 
Zwecke ist nach der herrschenden Lehre und Rechtsprechung widerrechtlich. ${ }^{34}$ Der Auschluss rein dividendenstrebiger Zielsetzungen ermöglicht es, Erfolg anders als nur nach Maßgabe des Reingewinns zu messen. Wichtiger ist aber, dass Kapitalgewinninteressen keine dominierende Stellung einnehmen und somit unternehmerischer Spielraum für die Verwirklichung auch anderer Zielsetzungen verbleibt. ${ }^{35}$ Die Genossenschaft eignet sich somit dann als Rechtsträger, wenn eine mehrdimensionale Nutzenschaffung und nicht lediglich „Gewinn“ und „Dividende" im Zentrum stehen sollen.

\subsection{Identitätsprinzip}

In enger Verbindung mit der genossenschaftstypischen Mitgliederförderung und der demokratischen Grundstruktur steht das Identitätsprinzip, d. h. das personale Gleichsein von Trägern der demokratischen Entscheidungsfindung und Kontrolle, Nutzern (Abnehmern, Lieferanten) und Kapitalgebern. ${ }^{36}$ Danach sind Genossenschafter, anders als beim Grundtypus einer Kapitalgesellschaft, nicht nur als Kapitalgeber, die lediglich ein Kapitalgewinninteresse verfolgen, an der Gesellschaft beteiligt, sondern sie sind trägerschaftlich, leistungs- und finanzwirtschaftlich als Mitglieder, Kunden, Arbeitnehmer, Zulieferer, Abnehmer oder in anderer Weise meist mehrfach mit der Genossenschaft verbunden. ${ }^{37}$ Auch diese Überlagerung der Funktionen und Rollen der Mitglieder begünstigt u. E. die Entwicklung einer differenzierten und nicht auf die schlichte Profitmaximierung ausgerichteten unternehmerischen Tätigkeit.

Bemerkt sei, dass das Nichtmitgliedergeschäft und auch die Gemeinnützigkeit vor diesem Hintergrund atypisch erscheinen, weil damit das Identitätsprinzip durchbrochen wird. Zumindest nach schweizerischer Praxis gelten aber weder das Nichtmitgliedergeschäft noch die Gemeinnützigkeit als widerrechtlich. ${ }^{38}$ Selbst die

${ }_{33}$ Zum Begriff der Dividendenstrebigkeit vgl. Forstmoser, P./Taisch, F./Troxler, T./ D'Incà-Keller, I. (Fn. 13), S. 6-8.

34 BSK OR II-Baudenbacher, N 18 zu Art. 828 OR. Vgl. zum Ganzen auch Forstmoser, P./Taisch, F./Troxler, T./D'Incà-Keller, I. (Fn. 13), S. 6-8 und S. 26-29.

35 Vgl. Taisch, F./Jungmeister, A./Troxler, T./D'Incà-Keller, I. (Fn. 17), S. 24.

36 Vgl. Beuthien, V.: Genossenschaftsgesetz mit Umwandlungs- und Kartellrecht sowie Statut der Europäischen Genossenschaft, 15. Aufl., München 2011, § 1 N 8.

37 Vgl. hierzu auch Ringle, G.: Wettbewerbsvorteile durch Nutzung spezifischer Differenzierungspotentiale der genossenschaftlichen Kooperationsform, in: Münkner, H.-H./Ringle, G. (Hrsg.): Alleinstellungsmerkmale genossenschaftlicher Kooperation Der Unterschied zählt, Göttingen 2008, S. 34. Vgl. auch Art. 852-858 OR, welche die Rechte und Pflichten der Genossenschafter regeln.

38 Botschaft des Bundesrates an die Bundesversammlung zu einem Gesetzesentwurf über die Revision der Titel XXIV bis XXXIII des schweizerischen Obligationenrechts, vom 21. Februar 1928, BB1 1928 I 205, 287, 301. 
unterschiedslose Gleichbehandlung von Mitgliedern und Nichtmitgliedern im sogenannten Zweckgeschäft, wie es bei den großen Konsumgenossenschaften mitunter der Fall ist, oder die rein gemeinnützige Tätigkeit, dürfen aufgrund der seit Jahrzehnten geltenden und unwidersprochenen Praxis nunmehr als gewohnheitsrechtlich anerkannt betrachtet werden. ${ }^{39}$

\subsection{Prinzip der offenen Tür}

Die Genossenschaft ist nach dem Gesetzeswortlaut von Art. 828 Abs. 1 OR eine als Körperschaft organisierte Verbindung einer nicht geschlossenen Zahl von Personen oder Handelsgesellschaften. Die Umsetzung dieser offenen Struktur wird dadurch ermöglicht, dass das Grundkapital, sofern überhaupt eines besteht, zwingend variabel sein muss. ${ }^{40} \mathrm{Neu}$ eintretende Mitglieder können somit ohne die formellen Hürden der Kapitalerhöhung jederzeit Anteilscheine zeichnen $;^{41}$ bestehende Mitglieder können aus der Genossenschaft austreten und ihren Anteilschein verfallen lassen, ohne dass ein Kapitalherabsetzungsverfahren durchgeführt oder ein Käufer für die Anteilscheine gefunden werden muss. ${ }^{42}$ Diese offene und variable Mitgliedschafts- und Kapitalstruktur kann sich als vorteilig erweisen, wenn ein Unternehmen an eine große und allenfalls noch nicht bestimmte Vielzahl von Stakeholdern übertragen werden soll.

\subsection{Gemeinsame Selbsthilfe und Solidarität}

Nach der Vorstellung des historischen Gesetzgebers sollte die Förderung der Mitglieder (oder Dritter) primär durch die gemeinsame Selbsthilfe erreicht werden. ${ }^{43}$ Die Mitglieder sollen ihre wirtschaftlichen Interessen durch ihre persönliche Mitwirkung und durch geeignete Beiträge zur Genossenschaft selbst fördern. Die Beiträge können freilich unterschiedlich ausfallen, solange das Prinzip der relativen Gleichbehandlung nicht verletzt wird. ${ }^{44}$ Die bisherige Praxis des Bundesgerichts

${ }_{39}$ Vgl. zum Ganzen Forstmoser, P./Taisch, F./Troxler, T./D’Incà-Keller, I. (Fn. 13), S. 13,32 .

$40 \quad$ Vgl. Meier-Hayoz, A./Forstmoser, P. (Fn. 10), § 19 N 33 f. Vgl. Art. 828 Abs. 2 OR.

41 Sie sind gesetzlich sogar dazu verpflichtet, wenn die Genossenschaft Grundkapital aufweist. Vgl. Art. 853 Abs. 1 OR.

${ }^{42}$ Vgl. BSK OR II-Schwartz, N 9 zu Art. 842 OR. Vgl. 842-851und 864-874 OR.

43 Vgl. Art. 828 Abs. 1 OR. Der Selbsthilfegedanke findet seinen Ursprung in den im 19. Jahrhundert als Genossenschaften gegründeten „Notgemeinschaften“. Vgl. hierzu Dubach, W.: Das Recht auf Rückvergütung und Preisnachzahlung in der Genossenschaft, Diss. Zürich, Luzern 1932, S. 18; Egger, A.: Revision des Genossenschaftsrechtes, in: ZSR 41/1922, S. 109a.

${ }_{44}$ Vgl. hierzu Art. 854 OR. 
hat das Selbsthilfeprinzip allerdings vollständig ihres Gehalts entleert. ${ }^{45}$ Selbst die völlig beitragslose Genossenschaft gilt als zulässig. In der Tat finden sich denn auch zahlreiche Genossenschaften in der Schweiz, bei denen die Selbsthilfe der Mitglieder vollständig in den Hintergrund getreten ist. ${ }^{46}$

Mit der gemeinsamen Selbsthilfe wird aber auch die Solidarität und Verbundenheit der Mitglieder zum Ausdruck gebracht, was sich vor allem vor dem historischen Hintergrund verdeutlicht: Auslöser für die Gründung von Genossenschaften waren im 19. und frühen 20. Jahrhundert meist Mangel- und Notsituationen sowie der Wille Gleichbetroffener, sich gemeinsam selbst zu helfen ${ }^{47}$ Der Solidaritätsgedanke findet sich nicht nur im Begriff der gemeinsamen Selbsthilfe, sondern auch im Prinzip der offenen Tür und in der relativen Gleichbehandlung der Mitglieder. ${ }^{48}$ Mit dem Willen zur gemeinsamen Selbsthilfe wird gleichzeitig zum Ausdruck gebracht, dass auf Hilfe von außen, sei es vom Staat oder von Dritten, verzichtet wird. $^{49}$

\subsection{Kapitalbeschaffung}

Die Genossenschaft des Schweizerischen Obligationenrechts eignet sich weniger als die Aktiengesellschaft zur Eigenkapitalbeschaffung. ${ }^{50}$ Sie kann zur Eigenfinanzierung zwar Anteilscheine ausgeben. Auf der Risikoseite weisen diese aber ein aktienähnliches Profil auf, während das Gewinnpotenzial durch die bei Nichtkre-

45 Das Bundesgericht hat diese Praxis bereits im BGE 93 II 30 explizit geschützt. Vgl. hierzu Forstmoser, P./Taisch, F./Troxler, T./D’Incà-Keller, I. (Fn. 13), S. 13 m. w. N. in dortiger Fn. 86. In einem neueren Entscheid führte das Bundesgericht allerdings in einem obiter dictum aus, dass der Körperschaftszweck unter persönlicher Mitwirkung der Genossenschafter zu erreichen sei. Ein solches Verständnis der gemeinsamen Selbsthilfe entspricht dem Willen des historischen Gesetzgebers, steht aber im Widerspruch zur seit Jahrzehnten tolerierten Praxis der völlig beitragslosen Genossenschaft. Hierzu ausführlich Taisch, F./Troxler, T.: Mindestmitgliederzahl bei Genossenschaften, Bundesgericht, I. Zivilabteilung, Urteil vom 25. Mai 2012 i.S. Genossenschaft X. c. B. und C. (4 A_729/ 2011, BGE 138 III 407), in: AJP 11/2012, S. 13. Sollte damit eine Praxisänderung eingeläutet werden, hätte dies weitreichende, negative Auswirkungen auf die heute stark arbeitsteilig strukturierten Genossenschaftsunternehmen.

46 Vgl. Forstmoser, P./Taisch, F./Troxler, T./D'Incà-Keller, I. (Fn. 13), S. 29.

47 Vgl. Reymond, J.-A./Trigo Trindade, R. (Fn. 21), S. 7.

48 Vgl. Art. 828 und 854 OR.

49 Vgl. zum Ganzen auch Forstmoser, P./Taisch, F./Troxler, T./D’Incà-Keller, I. (Fn. 13), S. 11-13.

50 Vgl. zum Ganzen Taisch, F./Troxler, T.: Eigenkapitalbeschaffung bei Genossenschaften, Möglichkeiten und Grenzen de lege lata, in: AJP 3/2013, S. 408 und passim. 
ditgenossenschaften gesetzlich zwingende Dividendenbegrenzung limitiert ist. ${ }^{51}$ Genau genommen darf die auf die Anteilscheine entfallende Quote des Reinertrags den landesüblichen Zinssatz für langfristige Darlehen ohne besondere Sicherheiten nicht übersteigen, während eine solche Begrenzung für Aktien nicht existiert. ${ }^{52}$ Diese Limitierung ist vor dem Hintergrund zu verstehen, dass mit der Genossenschaft die unmittelbare Förderung der Mitglieder in bestimmten wirtschaftlichen oder ideellen Interessen angestrebt werden soll. Gewinnausschüttungen gehen folglich denknotwendig zulasten einer optimalen unmittelbaren Förderung und sind deswegen zu limitieren. ${ }^{53}$

Die Kehrseite davon ist allerdings, dass Anteilscheine im Vergleich zu Aktien insbesondere vor dem Hintergrund des zwingenden Kopfstimmprinzips für Investoren unattraktiv sind, was die Beschaffung von Finanzkapital auf dem Kapitalmarkt stark erschweren kann. ${ }^{54}$ Es existieren zwar gangbare Ansätze, um die genannten und auch andere mit den Anteilscheinen verbundene Nachteile abzuschwächen. ${ }^{55}$ Allerdings besteht derzeit noch keine gefestigte Gerichtspraxis, weswegen die Implementierung dieser Ansätze mit entsprechenden Risiken verbunden ist. Im Ergebnis bedeutet dies, dass Genossenschaften mit der Ausgabe von Anteilscheinen wohl nur aus dem Kreis der Mitglieder, die ihrerseits primär an der unmittelbaren Förderung durch die Genossenschaft interessiert sind, Kapital beschaffen können. Dies kann einerseits vorteilhaft sein, limitiert aber die Möglichkeiten, wenn ein hoher Kapitalbedarf erforderlich ist. ${ }^{56}$

\subsection{Mindestmitgliederzahl}

Während eine $\mathrm{GmbH}^{57}$ oder eine $\mathrm{AG}^{58}$ von nur einer Person gegründet und betrieben werden kann, sind bei der Genossenschaft mindestens sieben Personen erforderlich. ${ }^{59}$ Das Bundesgericht hat in einem Entscheid vom 25. Mai 2012 (BGE 138 III 407 ff.) festgehalten, dass es sich bei dieser Mitgliederzahl um ein begriffsbestimmendes Merkmal der Genossenschaft handle. Sinkt die Mitgliederzahl unter sieben, hat die Genossenschaft gemäß Bundesgericht ihre Existenz verlo-

\footnotetext{
51 Vgl. zum Ganzen Forstmoser, P./Taisch, F./Troxler, T./D’Incà-Keller, I. (Fn. 13), S. 9.

Vgl. Art. 859 Abs. 3 OR.

Vgl. Forstmoser, P./Taisch, F./Troxler, T./D'Incà-Keller, I. (Fn. 13), S. 7 f.

Vgl. Taisch, F./Troxler, T. (Fn. 45), S. 408.

Vgl. Taisch, F./Troxler, T. (Fn. 45), passim.

Vgl. hierzu und zum Ganzen Taisch, F./Troxler, T. (Fn. 45), S. 408 f. und passim.

Art. 775 OR.

Art. 625 OR.

Vgl. Art. 831 Abs. 1 OR.
} 
ren. ${ }^{60}$ Die u. E. verfehlten Erwägungen des Bundesgerichts müssen bis zu einer allfälligen Korrektur als geltende Praxis hingenommen werden. ${ }^{61}$ Die vergleichsweise sehr hohe Mindestmitgliederzahl ${ }^{62}$ kann die Verwendung der Genossenschaft als Nachfolgeoption erschweren oder verhindern, wenn nicht genügend Personen zur Gründung oder für den Betrieb einer Genossenschaft vorhanden sind.

\subsection{Keine rechtsgeschäftliche Übertragung der Mitgliedschaft}

Eine weitere Besonderheit der Genossenschaft besteht darin, dass die Mitgliedschaft im Grundsatz rechtsgeschäftlich nicht übertragbar ist. ${ }^{63}$ Der Erwerb der Mitgliedschaft erfolgt originär mittels schriftlicher Beitrittserklärung und in der Regel durch einen Aufnahmebeschluss der Verwaltung oder der Generalversammlung und kann - vorbehaltlich weniger Ausnahmen ${ }^{64}$ - nicht durch Singularsukzession erworben werden. ${ }^{65}$ Dies bietet in Verbindung mit dem zwingenden Kopfstimmprinzip einen äußerst effektiven Übernahmeschutz. ${ }^{66}$ Eine Genossenschaft kann, abgesehen von einigen theoretisch denkbaren, praktisch aber nur schwer realisierbaren Angriffsmöglichkeiten, nicht von einem Investor gegen den Willen der Mitglieder übernommen oder kontrolliert werden. Im Unterschied zu anderen Rechtsformen, mit denen sich ähnliche Schutzvorkehrungen implementieren lassen, sind die genannten Elemente bei einer Genossenschaft gesetzlich zwingend vorgesehen.

\footnotetext{
$60 \quad$ BGE 138 III 407, E. 2.5.2.

61 Vgl. ausführlich hierzu Taisch, F./Troxler, T. (Fn. 45), S. 20.

62 Bei der AG und der GmbH genügt jeweils eine Person. Bei der einfachen Gesellschaft, Kollektiv- und Kommanditgesellschaft und beim Verein sind hingegen mindestens zwei Personen erforderlich. Dazu ausführlich Meier-Hayoz, A./Forstmoser, P. (Fn. 10), § 16 N 35 (AG); § 18 N 29, m. w. N. (GmbH); § 12 N 14 (einfache Gesellschaft); $\S 13$ N 11-13 (Kollektivgesellschaft); § 14 N 4 (Kommanditgesellschaft) und $§ 20$ N 8 (Verein).

${ }^{63}$ BSK OR II-Schwartz, N 1 zu Art. 849 OR.

${ }^{64}$ Vgl. Art. 840 OR. Ausnahmen: Erbschaft (Art. 847 Abs. 2 und 3 OR), Verknüpfung der Mitgliedschaft mit einem Vertrag (Art. 849 Abs. 3 OR) oder einem Grundstück (Art. 850 Abs. 2 OR).

${ }_{65}$ Vgl. Taisch, F./Troxler, T. (Fn. 45), S. 409-412.

${ }_{66}$ Vgl. CHK-Courvoisier, N 1-8 zu Art. 849 OR; BEK OR-Forstmoser, VII/4, N 32, 34 zu Art. 849.
} 


\section{Konstellationen, die für die Genossenschaft als Nachfolgemodell sprechen}

\subsection{Vorbemerkungen}

Ausgehend von den vorstehend nicht abschließend dargelegten Besonderheiten ${ }^{67}$ lassen sich bestimmte Kombinationen von Zielsetzungen und Interessen des Übergebers und des Übernehmers herleiten, die für die Genossenschaft als Nachfolgeoption sprechen. Daneben existieren andere Zielsetzungen, für die sich die Genossenschaft nicht eignet. Hierzu gehört unter anderem die Absicht des Übernehmers, eine möglichst hohe Rendite in Form von Gewinnausschüttungen zu erzielen. ${ }^{68}$ Gleiches gilt, wenn angestrebt wird, das Unternehmen alleine zu beherrschen und zu kontrollieren, ${ }^{69}$ insbesondere, um das Unternehmen als Tochtergesellschaft in eine bestehende Konzernstruktur einzubinden. ${ }^{70}$

\subsection{Erhalt des bestehenden Netzwerks}

Klein- und mittelständische Unternehmen sind oft eng mit lokalen Märkten und Unternehmungen verwoben. ${ }^{71}$ Geschäfte werden in Vereinen oder durch Mund-zuMund-Propaganda angebahnt und abgeschlossen, vielfach direkt durch den Unternehmensinhaber - den „Patron“ - selbst. Eine ständige Präsenz und Beziehungspflege mit lokalen Behörden, Berufs- und Branchenverbänden sowie eine enge, meist persönliche Beziehung zu den Kunden, Lieferanten und Abnehmern dient dazu, das Angebot und die Unternehmensstruktur ständig an die lokalen Bedürfnisse und Gegebenheiten anzupassen. ${ }^{72}$ Mit dem Ausscheiden des Patrons aus der Unternehmung geht eine für das Unternehmen essenzielle Funktion verloren. ${ }^{73}$ Die erfolgreiche Weiterführung des Unternehmens dürfte aber nicht zuletzt von der Erhaltung des bestehenden Netzwerkes abhängen. Daran dürften sowohl

${ }_{67}$ Vgl. mit weiterführenden Hinweisen Taisch, F./Jungmeister, A./Troxler, T./D'IncàKeller, I. (Fn. 17), S. 29 f.

${ }_{68}$ Vgl. Taisch, F. (Fn. 8), Rz 1.

${ }_{69}$ Vgl. Taisch, F./Troxler, T. (Fn. 45), S. 17.

70 Vgl. zum Ganzen auch Gerber, W.: Die Genossenschaft als Organisationsform von Mittel- und Grossunternehmen, Diss. Bern 2003, S. 88-153; Taisch, F. (Fn. 8), Rz 80-165.

71 Vgl. Portmann, R.: Die Wahl der Rechtsform als betriebswirtschaftliches Problem für Klein- und Mittelbetriebe, Diss. Zürich 1987, S. 27-35.

72 Gl. M. Portmann, R. (Fn. 71), S. 36 f.

73 Gl. M. Hegi, R.: Menschlich-psychologische Aspekte, in: Hegi, R./Staub, L. (Hrsg.): Fortsetzung folgt... Unternehmensnachfolge in KMU erfolgreich gestalten, Zürich 2001, S. 23. 
der Unternehmer, der sein Lebenswerk sichern will, als auch die Übernehmer, die das Unternehmen weiterführen wollen, interessiert sein.

Durch die Überführung des Unternehmens in eine Genossenschaft mit Stakeholdern als Mitglieder ${ }^{74}$ wird eine für die eingebundenen Stakeholder neutrale Plattform geschaffen. Somit kann das bestehende Netzwerk verstärkt und die Rolle des Patrons zumindest teilweise ersetzt werden. Die offene und variable Mitgliedschafts- ${ }^{75}$ und Kapitalstruktur ${ }^{76}$ sowie das zwingende Kopfstimmprinzip ${ }^{77}$, das eine unausgewogene Berücksichtigung von Einzelinteressen bei der Entscheidfindung strukturell verhindert, lassen die Genossenschaft als geeignetes Gefäß erscheinen, um einerseits unterschiedliche Interessen, die von den Stakeholdern an das Unternehmen herangetragen werden, auszugleichen und um andererseits gleichgelagerte Interessen zu bündeln. ${ }^{78}$ Es scheint allerdings nicht einfach, wie die nachfolgenden Ausführungen zum Erhalt der Wertschöpfungskette verdeutlichen werden, alle teils divergierenden Interessen in die Genossenschaft einzubeziehen und gleichzeitig für alle Mitglieder einen Mehrwert zu schaffen. Aus diesem Grund dürfte das hier angedachte Konzept vor allem dann erfolgreich sein, wenn vorwiegend diejenigen Stakeholder-Gruppen aus dem Netzwerk des Unternehmens als Genossenschafter in die Genossenschaft eingebunden werden, die im Kern gleiche oder ähnliche Interessen verfolgen. Die Einbindung von Stakeholdern mit (völlig) divergierenden Interessen könnte allenfalls dann erfolgreich sein, wenn eine gegenseitige Abhängigkeit unter ihnen besteht und sie anerkennen, dass sich aufgrund dieser Abhängigkeit ihre individuellen Ziele nicht ohne die Berücksichtigung der Interessen der anderen Mitglieder verwirklichen lassen. Essenziell dürfte sodann auch der Wille der Mitglieder sein, mittels genossenschaftlicher Entscheidungsfindung eben diesen Interessenausgleich oder Kompromiss zu finden, und sich den entsprechenden Beschlüssen anschließend zu unterwerfen.

\subsection{Erhalt der Wertschöpfungskette und Einbindung von Stakeholdern in eine neutrale Unternehmensplattform}

Das Unternehmen kann im Laufe der Zeit eine sehr starke Stellung in der Zulieferer- oder Vertriebskette aufgebaut haben. Als Ergebnis entsteht eine enge Verflechtung verschiedener Stakeholder, wie Zulieferer oder Vertriebspartner, mit dem Unternehmen. Diese kann sehr effizient sein, weil für alle Stakeholder Skaleneffekte erreicht werden, die - im Idealfall fair - auf die verschiedenen Player

\footnotetext{
$74 \quad$ Vgl. zu den Einschränkungen sogleich nachfolgend.

75 Vgl. hierzu oben 2.4.

76 Vgl. hierzu oben 2.1. und 2.6.

77 Vgl. Art. 885 OR. Näheres hierzu oben 2.1.

78 Vgl. Münkner, H.-H./Tonnellier, H.-J./Siebert, J.: Ist die Genossenschaft als Wirtschaftsform noch wettbewerbsfähig?, Marburg 2004, S. 18.
} 
entlang der Wertschöpfungskette aufgeteilt werden. Bei einer Nachfolgeregelung besteht die Gefahr, dass das Unternehmen eine exklusivere Zusammenarbeit mit ausgesuchten Stakeholdern sucht. Durch die Überführung des Unternehmens in eine Genossenschaft mit Stakeholdern als Mitglieder könnte dem entgegengewirkt und die Stellung und Neutralität des Unternehmens innerhalb der Wertschöpfungskette erhalten werden. Die Beschränkung in der freien Gewinnverwendung ${ }^{79}$ schafft dabei die Garantie, dass die Dividendenmaximierung nie zum eigentlichen Ziel der Genossenschaft wird und dient somit der Fokussierung des Unternehmens auf die Realisierung bestimmter wirtschaftlicher Interessen der als Mitglieder eingebundenen Stakeholder. ${ }^{80}$

Die Weiterführung als Gemeinschaftsunternehmen dürfte vor allem dann erfolgreich sein, wenn die als Genossenschafter eingebundenen Stakeholder gleichgerichtete oder nicht stark divergierende Interessen verfolgen.$^{81}$ Denkbar wäre beispielsweise, dass Zulieferer das Unternehmen, welches bis anhin ihr Hauptabnehmer war, gemeinschaftlich als Absatzgenossenschaft weiterführen. Oder, dass die Kunden eines Unternehmens dieses als Konsumgenossenschaft erhalten wollen.

Gut denkbar wären in diesem Sinne auch Konstellationen, bei denen sich beispielsweise Zulieferer und Arbeitnehmer als Genossenschafter einbinden. Die entsprechenden Interessenlagen sind zwar nicht deckungsgleich, sie erscheinen aber immerhin kompatibel.

Schwieriger erweist sich demgegenüber eine erfolgreiche Einbindung von Stakeholdern mit weitgehend gegenläufigen Interessen. Es ist beispielsweise höchst anspruchsvoll, Zulieferer und Abnehmer eines Unternehmens in die Lage zu versetzen, dieses gemeinsam als Genossenschaft erfolgreich weiterzuführen. Die Einbindung solcher weitgehend gegenläufiger Interessen dürfte u. E. aber dann möglich sein, wenn die Verwaltung der Genossenschaft eine umsichtige, faire Preispolitik fährt und gleichzeitig eine interessenausgleichende Unternehmenspolitik, mit Blick auf den spezifischen Zweck der Genossenschaft, in den Statuten verankert wird.

\subsection{Krisenbewältigung im Rahmen der Nachfolge}

Im Rahmen einer Unternehmensnachfolge gestattet die Rechtsform der Genossenschaft Mitarbeitern, die ihre Arbeitsleistung einbringen und gleichzeitig Eigentümer und Manager sind, in die demokratische Entscheidungsfindung einzubeziehen sowie der gesamten Belegschaft eine weitreichende Beteiligung am Unternehmen zu ermöglichen. Die Einbindung der Mitarbeiter könnte sich besonders

\footnotetext{
79 Vgl. hierzu oben 2.6 und Art. 859 OR.

80 Vgl. hierzu oben 2.2.

81 Vgl. hierzu oben 3.2.
} 
dann als vorteilig erweisen, wenn nicht nur die Nachfolge, sondern auch die Bewältigung einer Unternehmenskrise ansteht. ${ }^{82}$ Auf diesem Weg könnte nämlich die Akzeptanz für Veränderungen und die Bereitschaft für innovative Ideen, wie z. B. Gehaltsverzichte zur Überbrückung der Krise sowie zum Verzicht auf eigene Ansprüche erhöht werden. Dies erscheint vor allem dann möglich, wenn mittel- und langfristig nach der Übernahme des Unternehmens und der Überbrückung der Krise, für die Mitarbeiter proportional zu ihrer Arbeitsleistung Gewinnmöglichkeiten bestehen.

In dieser Konstellation kommt der Selbsthilfecharakter ${ }^{83}$ der Genossenschaft besonders zum Vorschein. ${ }^{84}$ Die Mitarbeiter bringen ihre gesamte Arbeitskraft in den Geschäftsbetrieb ein und erhalten eine angemessene Beteiligung am Unternehmen. ${ }^{85}$ Jeder Mitarbeiter hat somit unmittelbaren Anteil am Erfolg, ist aber auch genauso vom Misserfolg betroffen. Dies kann sowohl das Unternehmertum als auch die Flexibilität und Effizienz der Beschäftigten fördern. Durch die Wahl der Genossenschaft als Rechtsträgerin kann sodann im Unterschied zu anderen Rechtsformen sichergestellt werden, dass zukünftige Gewinne nicht nach Maßgabe der Kapitalbeteiligung verteilt werden, sondern im Verhältnis zur Arbeitsleistung, die zum Gewinn beigetragen hat. Die direkte Förderung der wirtschaftlichen Interessen der Mitglieder kommt in dieser Konstellation im Erhalt bestehender bzw. in der Schaffung neuer Arbeitsplätze zum Ausdruck. Dieses Förderziel stellt zudem längerfristig die Weichen für bessere Arbeitsbedingungen.

Im Kern handelt es sich beim beschriebenen Modell um eine sogenannte Arbeiterproduktivgenossenschaft. ${ }^{86}$ Dabei ist allerdings zu bedenken, dass sich in der Schweiz Produktivgenossenschaften als nicht entwicklungsfähige Gebilde erwiesen haben. ${ }^{87}$ Dies ist primär auf Kapitalmangel, das Fehlen von Marktkenntnis und kaufmännischem Wissen unter den Genossenschaftern zurückzuführen. ${ }^{88}$ Bei der Umsetzung sind demzufolge Vorkehrungen zu treffen, welche diese bekannten Schwachstellen vermindern oder beheben können.

${ }_{82}$ Vgl. hierzu Hackner, K./Schönherr, K. W.: Unternehmensnachfolge im Mittelstand, Vom Mitarbeiter zum Unternehmer, Zürich 2007, S. 53.

83 Vgl. hierzu oben 2.5.

${ }^{84}$ Zur Produktivgenossenschaft als besonderer Typus ZHK OR-Gutzwiller, V/6/1, N 11 zu Art. 853 OR.

85 BEK OR-Forstmoser, VII/4, N 103 zu Art. 828 OR; Eisen, A. (Fn. 4), S. 37.

86 Vgl. hierzu auch Forstmoser, P./Taisch, F./Troxler, T./D’Incà-Keller, I. (Fn. 13), S. 27 f.; Höser, R.: Konkurrenzfähigkeit der Rechtsform Genossenschaft, Diss. Frankfurt am Main, Idstein 1989, S. 28.

87 BEK OR-Forstmoser, VII/4, ST N 170-173.

88 Vgl. Höser, R. (Fn. 89), S. 30-32. 


\section{5 Übernahmeschutz}

Eine KMU ist zumeist auch das „Lebenswerk“ des Unternehmers. Die Unternehmung wurde von ihm gegründet und ist von seinen Ideen, Wertvorstellungen und seiner Persönlichkeit geprägt. ${ }^{89}$ Das Kopfstimmprinzip und der Ausschluss der rechtsgeschäftlichen Übertragung ${ }^{90}$ bieten hohe Gewähr dafür, dass eine Genossenschaft nicht von finanziellen Investoren übernommen werden kann. Direktinvestoren, Private Equity Gesellschaften oder Fonds sind in der Regel nicht unmittelbar am operativen Geschäft des Unternehmens interessiert, sondern primär an einer möglichst guten „Verzinsung“ des investierten Kapitals und veräußern im Regelfall das Übernahmeobjekt innerhalb von 5 Jahren wieder. ${ }^{91}$ Die Genossenschaft als Nachfolgelösung ist demnach für diejenigen Unternehmer interessant, die primär ihr Lebenswerk langfristig erhalten und es daher von der Übernahme durch Finanzinvestoren schützen wollen. Unter diesen Voraussetzungen besteht vielfach auch die Bereitschaft des Unternehmers, das Unternehmen zu einem günstigen Preis zu verkaufen respektive einen Discount zu gewähren. Im Falle eines genossenschaftlichen Employee-Buy-Outs ließe sich ein Preisnachlass beispielsweise damit begründen, dass die neuen Eigentümer bekannt und mit den Geschäftsprozessen vertraut sind und darüber hinaus die Vision des Gründers teilen. ${ }^{92}$

\section{Zusammenfassung}

Die Genossenschaft eignet sich als Nachfolgeoption dann, wenn die Nachfolger keine dividendenstrebigen Zwecke verfolgen, oder wenn es eine Vielzahl unterschiedlicher Stakeholder an das Unternehmen zu binden gilt und dabei die Dominanz von Partikulärinteressen begrenzt werden soll. Die Genossenschaft kann ein Mittel sein, um das bestehende Netzwerk, in welches das Unternehmen eingebunden ist und oftmals vom Unternehmensinhaber selbst gepflegt wird, der nächsten Generation zu erhalten. Auch die Sicherung der bestehenden Wertschöpfungskette, die Einbindung von Stakeholdern in eine neutrale Unternehmensplattform und die Sicherung des „Lebenswerks“ vor der Übernahme durch Finanzinvestoren sind denkbare Zielsetzungen, die für die Genossenschaft als Nachfolgeoption sprechen. Ungeeignet ist die Genossenschaft demgegenüber, wenn das Unternehmen in eine bestehende Konzernstruktur eingebunden werden soll, wenn die alleinige Kontrolle angestrebt wird oder sich das Interesse der Übernehmer auf die Dividenden beschränkt.

\footnotetext{
$89 \quad$ Vgl. Hegi, R. (Fn. 73), S. 23.

90 Zu den Ausnahmen siehe oben Fn. 64.

${ }_{91}$ Vgl. Halter, F./Schröder, R. (Fn. 18), S. 68.

92 Vgl. hierzu Halter, F./Schröder, R. (Fn. 18), S. 69.
} 